Management international

Gestiòn Internacional

International Management

\title{
Combler le " vide institutionnel " : Le comportement social et les performances des entreprises familiales comparés à ceux des autres entreprises des marchés émergents dans le secteur des hautes technologies.
}

\author{
Danny Miller, Jangwoo Lee, Sooduck Chang et Isabelle Le Breton-Miller
}

Volume 14, numéro 1, automne 2009

L'entrepreneuriat familial, un champ en devenir

Family entrepreneurship: a constantly evolving field

El empresariado familiar, un campo en desarrollo

URI : https://id.erudit.org/iderudit/039140ar

DOI : https://doi.org/10.7202/039140ar

Aller au sommaire du numéro

Éditeur(s)

HEC Montréal et Université Paris Dauphine

ISSN

1206-1697 (imprimé)

1918-9222 (numérique)

Découvrir la revue

Citer cet article

Miller, D., Lee, J., Chang, S. \& Le Breton-Miller, I. (2009). Combler le « vide institutionnel ": Le comportement social et les performances des entreprises familiales comparés à ceux des autres entreprises des marchés émergents dans le secteur des hautes technologies. Management international / Gestiòn Internacional / International Management, 14(1), 67-83.

https://doi.org/10.7202/039140ar
Résumé de l'article

Les entreprises familiales (EF) sont réputées prendre un soin particulier de leurs employés, avec l'objectif de créer une " communauté » interne très soudée. Leurs relations et contacts avec leurs partenaires extérieurs seraient aussi plus approfondis et personnels. Ces deux attitudes favoriseraient la viabilité d'une entreprise qui doit faire vivre la famille propriétaire, ainsi que les générations suivantes. Ces liens sociaux nous paraissent pouvoir compenser le manque de capital, de produits et d'infrastructure propre aux économies émergentes dynamiques. Cette étude, menée dans un secteur très compétitif de marché émergent, les entreprises de haute technologie en Corée, examine trois points majeurs :

(1) Les relations internes et les liens avec l'extérieur sont plus développés dans les EF que dans les autres. (2) Ces relations améliorent la performance des secteurs de haute technologie dans les marchés émergents, pour lesquels il est indispensable, du fait de leur nature compétitive complexe et évolutive, de disposer d'une expertise pointue et d'un capital social à l'intérieur comme à l'extérieur de l'entreprise. (3) Les performances des EF sont plus dépendantes de ces relations communautaires et de ces liens avec l'extérieur que celles des autres entreprises, parce que dans ce cadre personnel et intime, les employés et les partenaires extérieurs ont généralement tendance à récompenser la générosité, ou au contraire à punir l'égoïsme, d'une famille propriétaire qu'ils voient à l'oeuvre de leurs propres yeux. Nos observations empiriques confirment la plupart de ces hypothèses de façon significative.
Tous droits réservés ㅇ Management international / International Management / Gestión Internacional, 2009
Ce document est protégé par la loi sur le droit d'auteur. L'utilisation des services d’Érudit (y compris la reproduction) est assujettie à sa politique d'utilisation que vous pouvez consulter en ligne.

https://apropos.erudit.org/fr/usagers/politique-dutilisation/ 


\section{Combler le «vide institutionnel» : Le comportement social et les performances des entreprises familiales comparés à ceux des autres entreprises des marchés émergents dans le secteur des hautes technologies.}

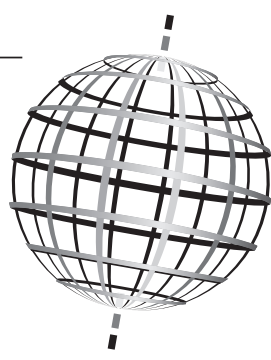

DANNY MILLER

HEC Montréal et Faculté de gestion

de l'Université d'Alberta

\author{
JANGWOO LEE \\ Université de Kyungopook, \\ Corée
}

\author{
SOODUCK CHANG \\ Université Hannam \\ de Corée
}

\begin{abstract}
ISABELLE LE BRETON-MILLER
HEC Montréal et Faculté de gestion de l'Université d'Alberta
\end{abstract}

\section{RÉSUMÉ}

Les entreprises familiales (EF) sont réputées prendre un soin particulier de leurs employés, avec l'objectif de créer une «communauté» interne très soudée. Leurs relations et contacts avec leurs partenaires extérieurs seraient aussi plus approfondis et personnels. Ces deux attitudes favoriseraient la viabilité d'une entreprise qui doit faire vivre la famille propriétaire, ainsi que les générations suivantes. Ces liens sociaux nous paraissent pouvoir compenser le manque de capital, de produits et d'infrastructure propre aux économies émergentes dynamiques. Cette étude, menée dans un secteur très compétitif de marché émergent, les entreprises de haute technologie en Corée, examine trois points majeurs :

(1) Les relations internes et les liens avec l'extérieur sont plus développés dans les EF que dans les autres. (2) Ces relations améliorent la performance des secteurs de haute technologie dans les marchés émergents, pour lesquels il est indispensable, du fait de leur nature compétitive complexe et évolutive, de disposer d'une expertise pointue et d'un capital social à l'intérieur comme à l'extérieur de l'entreprise. (3) Les performances des EF sont plus dépendantes de ces relations communautaires et de ces liens avec l'extérieur que celles des autres entreprises, parce que dans ce cadre personnel et intime, les employés et les partenaires extérieurs ont généralement tendance à récompenser la générosité, ou au contraire à punir l'égoïsme, d'une famille propriétaire qu'ils voient à l'œuvre de leurs propres yeux. Nos observations empiriques confirment la plupart de ces hypothèses de façon significative.

Mots clés : entreprise familiale, direction des ressources humaines (DRH), relations entre organisations, lacunes institutionnelles, capital social, marché émergent.

\begin{abstract}
We argue that family businesses (FBs) will tend to treat their employees with unusual consideration to form a cohesive internal "community". They are also expected to develop deeper, more extensive "connections" or relationships with outside stakeholders. Both behaviors should increase the viability of a business intended to support an owning family and its later generations. Such social linkages, we believe, may compensate for the lack of capital, product and labor institutional infrastructures in dynamic emerging economies. This survey study of a most challenging emerging market sector, namely, Korean high technology businesses, largely supports these expectations.
\end{abstract}

Keywords: Family firms, human resource management (HRM), inter-organizational relationships, institutional gaps, social capital, emerging markets
$\mathrm{N}$ otre premier point consistera à démontrer que les entreprises familiales (EF par la suite), plus enclines à développer des relations étroites avec leurs employés et leurs partenaires extérieurs, sont plus performantes dans les secteurs les plus imprévisibles des marchés émergents. En effet, ces liens se révèlent particulièrement utiles car ils comblent ce que Khanna et Palepu (1997) ont désigné comme un «vide institutionnel» propre aux marchés du capital, des produits et du travail de ces économies. Deuxièmement, nous proposerons, en comparant les EF et les autres entreprises, des hypothèses quant à l'importance relative de ces relations, et à leurs implications en terme de performance, et les examinerons dans un échantillon d'entreprises coréennes de haute technologie. En conclusion, nous discuterons nos résultats.

* Première parution en langue anglaise in Journal of International Business Studies (2009), traduction et adaptation Annick Baudoin et Alain Bloch.
Les EF représentent environ la moitié du PNB des ÉtatsUnis, emploient plus de la moitié de la main-d'œuvre, et créent plus de $85 \%$ de tous les nouveaux emplois (Shanker et Astrachan, 1996). Bien que souvent de petite taille, elles constituent environ un tiers des «Fortune 500 » (Anderson et Reeb, 2003). Elles sont encore plus nombreuses en Asie, représentant plus de $60 \%$ des entreprises «mid-cap» cotées à Hong Kong, Singapour et en Corée du Sud (LaPorta, Lopes-de-Silanes, \& Shleifer, 1999). Pourtant les EF ont toujours une image la plupart du temps négative et sont encore mal comprises (Miller \& Le Breton-Miller, 2005).

Cela s'explique par une apparente contradiction apparue tout récemment. La littérature sur les EF a maintes fois souligné leurs défauts éventuels : problèmes de succession, népotisme, capital insuffisant, conflits familiaux et mana- 
gement professionnel défaillant (Chandler, 1990; Gersick, Davis, Hampton \& Lansberg, 1997; Miller, Le BretonMilller, Lester \& Cannella, 2007; Schulze, Lubatkin, Dino \& Buchholtz, 2001). Cependant, des études récentes montrent que certaines EF résistent mieux que leurs pairs, qu'elles obtiennent de meilleurs bénéfices et une meilleure valorisation de marché (Anderson \& Reeb, 2003; Mackie, 2001; Villalonga \& Amit, 2006). Malgré le rôle essentiel de ces EF dans l'économie, nous en savons encore très peu sur leurs ressources particulières, ou les avantages concurrentiels qui seraient à la source de leurs performances.

À notre avis, la confusion qui entoure les EF et leurs performances s'explique par cette idée ambiante erronée qu'elles sont engluées dans le passé. On les décrit traditionnelles, trop humaines, paternalistes dans leurs relations avec les employés (Bertrand \& Schoar, 2006; Chandler, 1990; Landes, 1949; Lazonick, 1986), beaucoup trop sentimentales, sujettes au copinage, et trop complaisantes dans leurs associations de longue date avec les partenaires extérieurs (Morck, Wolfenzon, \& Yeung, 2005). La majorité des théoriciens les ont donc cataloguées "entreprises du passé" - trop figées ou peu performantes, surtout dans des environnements dynamiques (Chandler, 1990; Morck et al, 2005).

D'un autre côté, certains chercheurs ont démontré que la cohésion propre aux cultures de clan, où les employés sont engagés à long terme et généreusement traités, constitue un atout essentiel dans la compétitivité des industries de l'avenir (Collins, 1995; Davis \& Meyer, 1998; Koch, 2007; Miller, 2003; Miller \& Le Breton-Miller, 2005). Il en va de même pour leurs relations courantes et leur coopération avec les parties prenantes ou partenaires extérieurs -- fournisseurs, conseillers et collaborateurs -- qui apportent les ressources indispensables à l'exploitation des projets innovants et permettent à l'entreprise de se concentrer sur ses compétences distinctives (Adler \& Kwon, 2002; Bubolz, 2001; Nahapiet \& Ghoshal, 1998). Il peut donc paraître paradoxal que ces EF considérées comme des "vestiges du passé" puissent se rendre utiles dans les industries de haute technologie de l'avenir.

C'est sans doute particulièrement vrai dans les marchés émergents où les relations sociales étroites que réussissent à développer certaines EF compensent les "vides institutionnels" que sont l'absence relative d'entreprises intermédiaires, de mécanismes de régulation et des dispositions assurant le respect des contrats. Khanna et Palepu (1997) ont démontré que ces vides peuvent handicaper les échanges économiques dans les marchés de capitaux, du travail comme des produits. Par ailleurs, Khanna et Palepu (1999, 2000) et Khanna et Rivkin (2000) ont montré que dans les économies émergentes comme la Corée du Sud et l'Inde, certains groupes sont plus performants que des sociétés indépendantes parce que les relations à l'intérieur de ces groupes pallient souvent le vide institutionnel en fournissant un meilleur accès aux ressources humaines, financières et technologiques. Nous montrerons que ces relations étroites des EF avec leurs partenaires aussi bien internes qu'externes à leur société ont un même effet positif. Plus spécifiquement, ces liens étroits avec les employés et les partenaires extérieurs pourvoyeurs de financement, de conseil professionnel et de formation peuvent créer un contexte institutionnel qui compense les insuffisantes structurelles dans les domaines politiques, sociaux et économiques des marchés émergents. Ceci est particulièrement vrai dans un environnement compétitif et hautement technologique, où de tels liens sont nécessaires pour accéder aux rares ressources humaines et financières et pour amortir les risques (Nonaka, 1994, 1995).

Les EF de l'industrie coréenne de haute technologie nous fournissent des exemples parfaits pour étudier le sujet. En premier lieu, elles représentent plus de la moitié de l'économie du pays (Nam, 2002). Deuxièmement, comme au Brésil, en Russie et en Chine, le dynamisme et le développement de l'économie sud-coréenne sont parmi les plus forts du monde, et le pays est devenu un terrain très favorable à la création d'entreprises de haute technologie dans l'informatique, les télécommunications et le matériel spécialisé (Lee \& Chang, 1999; Lee, Lee \& Pennings, 2001). Finalement, comme dans les pays mentionnés cidessus, il n'existe pas en Corée d'institutions aussi développées qu'en Amérique et en Europe (Choi, 2004; Lee \& Lee, 1994). Khanna et Palepu (1999) ont déjà soutenu que des conglomérats (chaebols) coréens tels que Daewoo et Samsung ont formé des groupes diversifiés afin, justement, de pallier les vides institutionnels des marchés de la finance, du travail et des produits. La Corée constitue donc un terrain parfait pour vérifier si des liens étroits internes et externes à l'entreprise contribuent réellement à combler le vide institutionnel (Park, 1982).

Nous allons démontrer que ces particularités des EF leurs relations étroites avec leurs employés et leurs partenaires extérieurs - dont il est dit qu'elles les limitent à des contextes stables - sont justement celles qui leur permettent d'être performantes dans les secteurs les plus évolutifs des marchés émergents. Nous proposerons quelques hypothèses sur la nature de ces relations, et sur leurs conséquences sur la performance, dans les EF et dans les autres firmes de haute technologie en Corée.

\section{Communauté et relations dans les EF des marchés émergents}

Quand les entreprises sont dirigées par des propriétaires appartenant à une même famille, pour laquelle la firme est source de sécurité, de réputation et de profits pour eux-mêmes et leurs descendants, les relations entre ces propriétaires et leur entreprise sont généralement extrêmement étroites (Arregle, Hitt, Sirmon, \& Very, 2007; Miller, Le Breton-Miller, \& Scholnick, 2008; Zahra, Hayton, \& Salvato, 2004). Ces chefs d'entreprise sont très conscients de l'enjeu majeur de leur réussite sur le long terme : for- 
tune familiale, statut de la famille dans la société, et même la possibilité pour elle de poursuivre une tradition respectée ou une mission dont elle est fière (Miller \& Le-Breton Miller, 2005). Leur relation à l'entreprise et souvent celles avec les employés et les partenaires, sont généralement plus durables, généreuses et inclusives (Arregle et al, 2007). À l'inverse, les propriétaires d'entreprises non familiales sont plus fortement motivés par des raisons économiques (Jacobs, 1991; Ward, 2004). Ils visent la performance, un succès financier à court ou moyen terme, et se soucient moins de partenariat à long terme et de relations à caractère personnel, et encore moins à caractère intime (James, 1999). Ces différences présumées peuvent avoir des conséquences importantes sur la différence de comportement observée entre EF et autres sociétés envers leurs employés et leurs partenaires extérieurs, et sur la manière dont à leur tour, ces derniers servent les intérêts de l'entreprise.

Ainsi, certains chercheurs avancent que si les propriétaires des EF construisent une "communauté" d'entreprise, c'est pour renforcer la poursuite des objectifs de la firme et accroître sa longévité (Guzzo \& Abbot, 1990; Miller \& Le Breton-Miller, 2005). La cohésion de la communauté se traduit par la loyauté envers les employés, le souci de leur bien-être au-delà des exigences purement légales ou administratives, et la nécessité de leur donner un métier sûr et satisfaisant. Ainsi se créent souvent avec les employés des liens étroits qui compensent la rareté en talents des marchés du travail dans les économies émergentes (Khanna \& Palepu, 1997).

On dit aussi que les EF semblent vouloir créer des "réseaux", c'est-à-dire constituer un véritable capital social, en nouant des relations durables avec une grande variété de partenaires extérieurs pourvoyeurs de l'entreprise (Arregle \& al., 2007; Bubolz, 2001; Miller \& Le Breton-Miller, 2005). Ces partenaires sont les indispensables fournisseurs de connaissance, de relations sociales et de capital financier à même de renforcer l'entreprise, d'assurer sa stabilité et de développer ses capacités (Adler \& Kwon, 2002; Hagel \& Singer, 1999; Nahapiet \& Ghoshal, 1998). De telles relations - alliances et joint ventures au lieu de négociations "one shot" - peuvent elles aussi contribuer à compenser le vide institutionnel dans le domaine des transactions financières, des produits, de la connaissance et des fournitures (Khanna \& Palepu, 1997, 1999). Ce capital social, fondé sur la confiance et sur la fidélité et assuré par la stabilité des relations, diminue le plus souvent les risques associés à un projet. En outre, former ces relations et en tirer profit est souvent plus facile aux propriétaires et managers des EF qu'aux autres, car non seulement ils sont motivés pour signer des engagements mais aussi en mesure de les honorer (Bubolz, 2001; Miller \& Le Breton-Miller, 2005; Saxton, 1997).

Malheureusement, aucune étude ne s'est encore penchée sur la prédominance dans les EF de ces communautés et de ces réseaux de relations, ni sur leurs conséquences sur la performance des sociétés de haute technologie dans les marchés émergents. C'est ce que nous allons faire ici.

\section{Hypothèses sur la communauté}

\section{Prédominance relative de la communauté dans les EF}

Les études sur l'engagement organisationnel envers les employés ("orgazinational commitment to employees" OCE) ont identifié une notion proche du concept de communauté défini par Miller \& Le Breton-Miller (2005). Ce concept d'OCE s'appuie sur la notion de "perception du soutien organisationnel", élaborée par Eisenberger, Huntington, Hutchison et Sowa (1986), plus tard approfondi et opérationnalisé par Lee \& Miller (1999) et Miller \& Lee (2001). En gros, ce concept définit la disposition de l'organisation à faire le maximum pour son personnel : elle pratique des salaires généreux, le partage des profits, veille à la sécurité des employés et même à leur satisfaction et à leur progression dans le travail. Certains chercheurs ont avancé que l'OCE suscite généralement en retour la loyauté des employés et les motive à travailler plus et plus intelligemment (Eisenberger et al., 1990; Lee \& Miller, 1999; Miller \& Lee, 2001; Moorman, Blakely \& Niehoff, 1998; O'Reilly \& Chatman, 1986). Malheureusement, l'OCE n'a guère été étudié à l'extérieur des économies développées, ou dans les environnements les plus concurrentiels d'aujourd'hui. Pourtant les entreprises des marchés émergents y ont probablement recours pour lutter contre la pénurie et les défauts du marché du travail (Khanna \& Palepu, 2000,2006; Nam, 2002). Tout comme les sociétés de haute technologie qui sont elles aussi confrontées à la compétition sur le plan des connaissances, car il leur est impératif d'attirer et de garder les meilleurs talents (Nonaka, 1994).

Comme nous l'avons déjà noté, alors que les entreprises non familiales tendent à adopter une attitude impersonnelle ou "professionnelle" en visant des objectifs financiers, les dirigeants d'EF sont des gestionnaires du long terme, soucieux des objectifs de leur société et de leur personnel - et ce sur plusieurs générations (Gersick et al.,1997; Ward, 2004). En effet, ils gèrent non seulement leur réputation et leur fortune, mais aussi celles de leurs enfants et des autres membres de la famille, acteurs actuels et futurs de la société. Pour eux, l'entreprise n'est pas seulement un moyen de gagner de l'argent, mais aussi un prolongement de la famille et de sa réputation dans la communauté, une façon d'assurer la vie de leurs enfants et des autres membres de leur famille, et de répondre à leurs aspirations de carrière (Miller et al., 2008). Ils sont donc impliqués corps et âme dans leur entreprise, soucieux de leurs employés, et prêts à investir généreusement pour eux (Allouche \& Amann, 1997; Reid \& Harris, 2002). C'est d'eux en effet que dépendent sa santé et sa créativité. En conséquence, les propriétaires d'EF veillent particulièrement à soigner leurs relations avec leurs salariés, c'est-à-dire à faire preuve d'un degré élevé d'OCE (Guzzo \& Abbott, 1990). 
Tout un ensemble d'éléments montrent l'importance majeure que de nombreuses $\mathrm{EF}$ américaines attachent à l'OCE pour renforcer la cohésion interne de leur communauté. Gersick \& al. (1997) mentionnent l'altruisme et la confiance comme facteurs essentiels de succès pour les EF. Dyer (1986) affirme que cette attitude «paternaliste» vise aussi les employés n'appartenant pas à la famille, et instaure dans l'entreprise un sentiment de stabilité propice à l'investissement personnel de chacun. Selon Allouche et Amann (1997) et Miller \& Le Breton-Miller (2005), les EF octroient des salaires exceptionnellement élevés, proposent de nombreuses formations et évitent les licenciements. Beehr, Drexler et Faulkner (1997), Donckels et Fröhlich (1991) et Guzzo et Abbott (1990) ont repris nombre de ces arguments. Nous proposons donc l'hypothèse suivante :

Hypothèse 1 : Dans les marchés émergents, le sens de la communauté, évalué par l'OCE, est plus forte dans les $\mathrm{EF}$ que dans les entreprises non-familiales.

Cette hypothèse ne fait pas l'unanimité. Certains chercheurs démontrent que les EF sont particulièrement pingres car privées d'accès aux capitaux, enclines au népotisme, aux conflits familiaux et au sentimentalisme, et qu'elles opèrent une discrimination contre les managers étrangers à la famille (voir la revue de ces articles chez Chandler, 1990; Morck et al., 2005; Schulze et al., 2001; Singell, 1997). Si c'est le cas dans certaines EF, on a montré que c'est extrêmement rare dans des environnements concurrentiels et évolutifs, c'est-à-dire ceux que nous étudions ici (Carney, 2005).

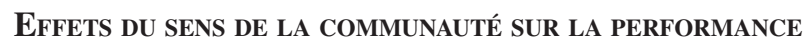

Dans le domaine de la haute technologie, où l'initiative et la créativité des employés sont primordiales pour la vitalité et la compétitivité de l'entreprise, l'OCE est essentiel à la performance. L'attention portée au personnel contribue à attirer et garder les meilleurs éléments (Eisenberger \& al., 1990; Lee \& Lee, 1994). Il faut des hommes de talent pour inventer et commercialiser de meilleurs produits, innover avec succès et trouver de nouveaux marchés (Galbraith, 2000). La stabilité du personnel associée à un climat d'entreprise positif préserve les savoirs de l'équipe, surtout les savoir-faire de l'entreprise, et les protège contre les concurrents - renforçant à son tour les processus d'innovation et de collaboration nécessaires en haute technologie (Barney \& Hansen, 1994; Nonaka, 1995). Lorsque cette réalité est ignorée ou implicitement niée, les dégraissages et les restructurations importantes auxquels beaucoup d'entreprises ont recours nuisent à leur propre force de travail (Miller \& Le Breton-Miller, 2005).

Nous nous attendons donc, comme déjà suggéré, à ce que le dévouement envers les employés soit payé de retour, suscitant ou augmentant chez eux un niveau de motivation et d'attachement affectif fort utile dans le monde de la haute technologie (O’Reilly \& Chatman, 1986; Orpen, 1995). En effet un personnel dévoué et motivé constitue une ressource rare, précieuse et inimitable permettant aux entreprises de mettre au point de puissante stratégie d'innovation (Barney \& Hansen, 1994; Lee \& Miller, 1999). Il permet également d'augmenter l'initiative et la collaboration parmi les décideurs - qualités essentielles au succès de l'innovation (Miller \& Lee, 2001).

Même si Lee \& Miller (1999) n'ont pas trouvé de réels effets concluants de l'OCE sur la performance, c'est qu'ils ne se sont pas concentrés sur les entreprises de haute technologie, où l'innovation, la créativité et la préservation des connaissances de l'entreprise sont primordiales. Or c'est dans ces contextes que nous attendons les effets bénéfiques de l'OCE sur la performance. En effet le sens de l'initiative des collaborateurs provoqué par l'OCE est particulièrement attendu dans les secteurs incertains de la haute technologie qui requièrent des structures organisationnelles à hiérarchie écrasée, des styles de management axés sur l'initiative des employés, et des systèmes où les décisions sont prises en collaboration pour renforcer l'esprit d'innovation nécessaire (Cooper, Willard, \& Woo, 1986; Duchesneau \& Gartner, 1990; Miller \& Lee, 2001).

Le contexte des marchés émergents peut aussi accrô̂tre la nécessité de l'OCE. En effet, selon Khanna et Palepu (1997, 1999, 2006), le vide institutionnel du marché du travail propre aux économies émergentes rend cruciale la stabilité des relations avec les employés de talent. Et du fait de l'absence d'organisations intermédiaires et de mécanismes assurant la sécurité juridique des contrats, s'assurer une main-d'œuvre stable, fiable et motivée est un véritable défi. C'est ainsi, par exemple, que pour y répondre, des entreprises coréennes mélangent éthique confucéenne et paternalisme pour créer un climat de bonne entente avec leurs employés, et obtenir en retour leur dévouement (Lee \& Lee, 1994; Shin, 1993). Dans ce cas, on considère que l'engagement de l'organisation envers ses employés, avec en retour le dévouement et la cohésion de la communauté, sont la pierre angulaire du succès de l'entreprise (Lee \& Miller, 1999). Ainsi l'OCE, sous forme de "culture paternaliste", est source d'avantages concurrentiels.

Hypothèse 2: La communauté, évaluée par l'OCE, contribue positivement à la performance dans les économies de marchés émergents.

D'autres études se sont penchées sur cette hypothèse, et l'ont soutenue, mais sans l'observer ni dans des contextes de haute technologie ni dans les marchés émergents, où l'OCE est essentiel du fait de l'importance de l'initiative des employés, de leur créativité et de leur intérêt pour une démarche innovatrice. Ces études n'ont pas non plus observé les petites EF, où une relation étroite et personnelle entre propriétaires et employés, source de confiance et de générosité réciproques, rend l'OCE particulièrement efficace. 


\section{L'AVANTAGE de la FAMILle dans la COMMUNAUté (OCE).}

Si l'OCE est bénéfique à toutes sortes d'entreprises de haute technologie (Lee \& Miller, 1999; Miller \& Lee, 2001), elle l'est surtout dans les EF où travaillent de nombreux membres de la famille et où se développent si souvent des relations personnelles entre les actionnaires-dirigeants et leur personnel (Ward, 2004). En effet, les employés constatent la générosité et la loyauté des membres d'une famille visible et concrète, et non pas d'une entité impersonnelle représentant l'entreprise, et leur attitude s'en trouve stimulée en retour. Un OCE généreux y émane d'une source humaine clairement identifiée, et les relations entre une famille bienveillante et des employés reconnaissants sont plus intimes et personnelles qu'ailleurs. Ces derniers constatent euxmêmes les efforts accomplis par la famille. Ayant souvent à faire directement à nombre de ses membres, ils apprennent à apprécier leur caractère et leur valeur, et s'ils les respectent véritablement, leur fidélité en est accrue. Autre atout, les actionnaires-dirigeants d'entreprise sont libres d'accorder des récompenses particulières de façon ponctuelle, c'est-à-dire d'ignorer la bureaucratie et les soucis financiers de court terme, et de se montrer très généreux envers des employés particulièrement méritants ou dans des circonstances exceptionnelles. De tels actes de libéralité engendrent souvent un rare dévouement. Habbershon \& Williams (1999) ont démontré que c'est exactement ce genre de ressources humaines et sociales qui sont les clés d'un avantage concurrentiel et de performances dans les EF, et qu'il est difficile de les reproduire dans de grandes sociétés impersonnelles axées uniquement sur le rendement à court terme.

Il faut cependant reconnaître qu'il peut exister des côtés négatifs au paternalisme des EF. Parmi eux, le favoritisme, le népotisme, la discrimination contre les cadres n'appartenant pas à la famille, l'absence de considération pour le mérite (pour ces critiques bien connues, voir Miller, Steier, \& Le Breton-Miller, 2003; Morck \& al., 2005; Schulze \& al., 2001; Singell, 1997). Ces failles font que lorsque l'OCE est faible, les $\mathrm{EF}$ le payent chèrement - ce qui en fait une variable encore plus importante pour les EF que pour les entreprises non-familiales.

Hypothèse 3 : L'influence de la communauté (OCE) sur la performance sera plus forte dans les EF de haute technologie des marchés émergents que dans les autres.

\section{Hypothèses relatives aux relations}

\section{Prédominance des relations dans les EF}

Il existe pour les entreprises deux modes d'interaction avec leurs partenaires extérieurs : les transactions et les relations (Arregle \& al., 2007; Saxton, 1997; Sirmon \& Hitt, 2003; Williamson, 1999). Dans le premier cas, l'échange se fait sur la base de la meilleure offre dans le contexte du marché, et l'objectif est purement économique. A l'inverse les relations impliquent des associations suivies qui construisent un capital social fondé sur des facteurs économiques mais aussi sur des éléments qui ne le sont pas. Elles représentent des liens plus étroits, plus stables et plus profonds.

Arregle et al. (2007) et Miller \& Le Breton-Miller (2005) démontrent que les EF ont une propension particulière à former de tels liens relationnels avec leurs partenaires extérieurs. Du fait de l'importance que les propriétaires d'EF accordent à la viabilité à long terme de l'entreprise, de leur volonté que leur société se transmette à leurs descendants familiaux, ils sont plus enclins que les autres à établir avec des parties prenantes extérieures les alliances susceptibles de leur apporter un savoir, des informations et un capital précieux (Miller \& Le Breton-Miller, 2005). La stabilité des relations extérieures des EF leur permet également de construire un capital social (Nam, 2002), qui les aide à traverser les passages difficiles souvent rencontrés dans les environnements instables de haute technologie (Saxton, 1997). En outre, elles contribuent à pallier les vides institutionnels si fréquents dans les marchés émergents (Khanna et Palepu, 1997, 1999). Enfin, les EF ont tendance à entretenir avec soin ces alliances susceptibles d'assurer l'avenir de leur société pour la génération suivante (Habbershon \& Williams, 1999; Sirmon \& Hitt, 2003).

Si les actionnaires dirigeants d'EF ont davantage intérêt à travailler avec des partenaires extérieurs, ils sont aussi plus aptes à le faire. Leur statut personnel fait d'eux une relation idéale pour les partenaires extérieurs, qui les considèrent comme des représentants stables et influents de leur entreprise, possédant le pouvoir de s'engager, la motivation pour le faire et les moyens de respecter leurs contrats sur le long terme. Les dirigeants propriétaires des EF restent souvent pendant des décennies à leur poste, ils ont à cœur les intérêts à long terme de leur société et de leur famille, et se comportent en conséquence dans leurs relations extérieures. Ces qualités facilitent les relations, et les stabilisent (Adler \& Kwon, 2002; Bubolz, 2001; Miller \& Le Breton-Miller, 2005, Sirmon \& Hitt, 2003).On peut donc en déduire que :

Hypothèse 4 : Dans les marchés émergents, les relations des EF de haute technologie avec leurs partenaires extérieurs sont plus développées que dans les entreprises non-familiales.

Soulignons que les EF ne formeront pas des types d'alliances qui risquent de menacer leur contrôle sur l'entreprise (Gomez-Mejia, Haynes, Nunez-Nickel, Jacobson \& Moyano-Fuentes, 2007). Elles favoriseront évidemment les relations qui leur apportent des ressources précieuses et des capacités de développement et refuseront celles qui les mèneraient à renoncer au contrôle financier de leur firme ou à compromettre leurs valeurs familiales (Miller \& Le Breton-Miller, 2005). 


\section{LES EFFETS DES RELATIONS SUR LA PERFORMANCE}

Étant donnée l'instabilité des environnements de haute technologie, ces relations suivies avec d'autres institutions et professionnels permettent souvent d'accroître les connaissances de l'organisation et de réduire les risques financiers (Adler \& Kwon, 2002). Pfeffer \& Salancik (1978) et leur école démontrent que la viabilité de ces sociétés dépend de leurs relations extérieures, qu'il leur est nécessaire de disposer de partenaires extérieurs pour s'assurer l'accès à l'information, aux talents, aux capitaux et aux clients. Et les besoins primordiaux des entreprises de haute technologie sont justement l'accès aux capitaux, aux connaissances, à la technologie et aux brevets. Elles les acquièrent grâce à de solides relations avec des partenaires extérieurs (Grant, 1996; Lee et al., 2001; Nahapiet \& Ghoshal, 1998; Nonaka, 1994).

Kanna \& Palepu $(1997,2000)$ ont montré que dans ces marchés émergents connus pour leur vide institutionnel, les groupes ayant conclu des engagements solides et durables réalisent de meilleures performances que les entreprises isolées. En effet, d'après eux, ces liens réduisent les coûts de transactions, créent une réputation et apportent un accès fiable aux ressources financières, humaines et technologiques, qui seraient autrement difficiles à obtenir (Khanna \& Palepu, 1997; Khanna \& Rivkin, 2000). Ces liens se nouent non seulement avec d'autres groupes, mais aussi avec les fournisseurs, partenaires et autres parties prenantes offrant du support en management et en technologie (Miller \& Le Breton-Miller, 2005).

Par ailleurs, pour certains de ces marchés émergents, on peut aussi soutenir que l'étendue géographique de ces liens les rend encore plus féconds. Ainsi les groupes trouvent un support à la diversification de leurs entreprises (Khanna \& Palepu, 1997, 1999) grâce à des liens régionaux, nationaux et surtout internationaux avec des partenaires, des clients et des fournisseurs, et ces liens sont de puissants moyens pour réduire les risques inhérents au contexte politique et économique local. Donc, l'étendue géographique de ces liens permet de diminuer le risque local.

Pour ces entreprises de haute technologie, les relations extérieures les plus importantes sont des accords de recherche avec des universités, et des sociétés et des organismes de recherche, ainsi qu'avec des consultants en management et en technologie, avec des agences gouvernementales, des spécialistes juridiques et techniques (Lee \& al.; 2001). Les joint ventures de recherche répartissent le risque, apportent des connaissances supplémentaires et facilitent les transferts de technologie, source importante d'innovation en Corée. Les liens avec des experts pointus apportent un savoir très spécialisé à même de soutenir les projets innovants et les efforts commerciaux. Les liens avec le gouvernement et les avocats peuvent contribuer à financer et développer des liens commerciaux (Lee \& al., 2001; Lee \& Chang, 1999). Ainsi :
Hypothèse 5 : Dans les marchés émergents, les relations et les liens avec des partenaires extérieurs favorisent la performance des entreprises de haute technologie.

\section{LeS AVANTAGES DE LA FAMILLE DANS LES RELATIONS}

De même que les relations entre employés et propriétaires favorisent un engagement réciproque, les relations durables avec des partenaires extérieurs construisent un capital social (Adler \& Kwon, 2002; Nahapiet \& Ghoshal, 1998). Les actionnaires dirigeants d'EF restent en général longtemps à leur poste, les responsables de la famille exerçant souvent pendant plus de vingt ans - contre trois ou quatre années pour les cadres d'autres entreprises (Miller \& Le Breton-Miller, 2005). Cette stabilité rehausse leur responsabilité vis-à-vis de leurs partenaires, qui savent qu'ils les trouveront toujours en poste pour répondre aux problèmes qui pourraient surgir. En retour, forts de cette crédibilité, les responsables d'EF se montrent dignes de cette confiance et agissent loyalement, ceci d'autant plus que la réputation et la fortune de leur société sont en jeu. Autre raison de leur fidélité à leurs engagements, ils résistent mieux aux fortes pressions ou aux conseils d'administration à courte vue. Tout ceci fait des EF des partenaires enviables - et donc plus aptes à susciter une générosité réciproque et une coopération de bon aloi chez leurs partenaires extérieurs. On ne sera pas surpris de résultats d'études prouvant que les EF ont tout à gagner à créer un capital social : il constitue pour eux un socle pour toutes sortes d'alliances avec l'extérieur (Arregle et al., 2007; Bubolz, 2001; Nahapiet \& Ghoshal, 1998; Sirmon \& Hitt, 2003).

L'entreprise restant solidement en la possession et sous la direction de la famille, les responsables familiaux peuvent aussi compter récolter les fruits de leurs relations pour les années à venir. Le concept de capital social est donc essentiel ici, les relations poursuivies sur le long terme engendrant la confiance et la bonne volonté et réduisant les coûts de transaction (Adler \& Kwon, 2002; Bubolz, 2001; Nahapiet \& Ghoshal, 1998). D’ailleurs, les relations se poursuivent souvent de génération en génération. Cela s'est produit chez J.P. Morgan et Bechtel, où, dans le saint des saints d'associations très fermés et de clubs élitistes, les descendants de la famille ont été présentés par leurs aînés aux riches et aux puissants (Miller et Le Breton-Miller, 2005). La confiance et la crédibilité acquise par une génération ont donc été transmises à la suivante (Gomez-Mejia, Nunez-Nickel \& Gutierrez, 2001). Les retombées potentielles à long terme de ce capital social expliquent également pourquoi les EF peuvent particulièrement bénéficier de ces relations avec des partenaires fidèles.

En revanche, dans des entreprises où les dirigeants restent brièvement en poste, le risque est grand de céder au désir d'une performance à court terme et d'agir par opportunisme, préférant une bonne affaire à la stabilité d'une relation. Les hauts responsables ont moins intérêt à proté- 
ger la réputation de l'entreprise, et à veiller à ce que des relations soient bénéfiques sur le long terme. Ils n'inspirent pas à leurs partenaires la même confiance ni la même crédibilité en affaires. De plus, ils ne sont pas toujours en mesure de prendre ces engagements exceptionnels qui appellent la réciprocité et rendent les relations particulièrement fécondes.

Hypothèse 6 : les relations et les liens avec des partenaires et des parties prenantes extérieurs ont, dans les marchés émergents, un effet plus positif sur la performance des EF que sur les entreprises non-familiales

Le tableau 1 résume nos hypothèses et leurs justifications.

\section{Méthode}

\section{DONNÉES}

Comme nous l'avons noté, et en accord avec Khanna \& Palepu (1997, 1999), nous avons choisi la Corée du Sud comme pays représentatif de marché émergent. Nous avons sélectionné comme sociétés cibles les 271 firmes indépendantes du Daegu High Tech Venture Guide List (2003), (celles situées dans les deux zones de haute technologie de la ville de Daegu). Nous avons déjà évoqué la pertinence du choix des firmes de haute technologie coréennes pour notre étude, et les deux zones de Daegu représentent une forte concentration de ce genre d'entreprises. Nous avons pris contact avec les directeurs ou les principaux dirigeants de ces entreprises dont 170 ont accepté de participer à notre étude (62\% de réponses positives).

Après avoir envoyé par courrier un questionnaire aux dirigeants ayant accepté de participer à cette enquête, nous les avons interrogés par téléphone. Avec nos assistants de recherche, nous les avons aidés à compléter le questionnaire, éclaircissant les points restés obscurs pour eux. (par exemple, le sens et l'importance des transferts de technologie). Nous les avons assurés que leurs réponses resteraient totalement confidentielles. Notre échantillon final comptait 52 entreprises d'informatique ou entreprises connexes (30,6\%), 40 fabricants d'équipement de communication (23,5\%), 36 fabricants de matériels spécialisés $(21,2 \%)$ et 42 firmes de composants électroniques (24,7\%). (Voir l'appendice A). Afin de prévenir le biais de méthode de variance commune, nous avons obtenu, pour 35 de ces firmes choisies au hasard, deux réponses indépendantes de leurs hauts dirigeants. Cela nous a permis d'évaluer le degré de concordance entre les répondants (fidélité inter-répondants) dont nous rendons compte ci-dessous.

\section{Mesures}

Nous avons mesuré nos variables en utilisant des échelles déjà éprouvées par d'autres chercheurs. Selon celles de La Porta \& al. (1999), de Miller \& Le Breton-Miller (2005), sont classées EP les entreprises où les actionnaires dirigeants énoncent que la famille possède plus de parts que

\section{TABLEAU 1}

\section{Résumé des hypothèses et justifications}

\begin{tabular}{l|l|l}
\hline & \multicolumn{1}{c|}{ Communauté (OCE) } & \multicolumn{1}{c}{ Relations } \\
\hline $\begin{array}{l}\text { Prédominance dans les EF } \\
\text { comparée aux entreprises } \\
\text { indépendantes }\end{array}$ & $\begin{array}{l}\text { Hypothèse 1 : Plus importante dans les } \\
\text { EF : l'avenir à long terme de la famille } \\
\text { motive l'investissement dans le capital } \\
\text { humain (Guzzo \&Abbott, 1990; Reid \& } \\
\text { Harris, 2002) }\end{array}$ & $\begin{array}{l}\text { Hypothèse 4 : plus poussées dans les EF : } \\
\text { le souci des générations suivantes motive la } \\
\text { formation de relations (Saxton, 1997; Simon } \\
\text { \& Hitt, 2003) }\end{array}$ \\
\hline $\begin{array}{l}\text { Effet sur la performance } \\
\text { dans les entreprises de haute } \\
\text { technologie des marchés }\end{array}$ & $\begin{array}{l}\text { Hypothèse 2 : Positive : la communauté } \\
\text { attire de bons éléments, motive } \\
\text { l'initiative et la collaboration; la loyauté } \\
\text { garde le capital de savoir-faire }\end{array}$ & $\begin{array}{l}\text { Hypothèse 5 : Positives: les relations } \\
\text { apportent le capital, le savoir, la technologie } \\
\text { et l'information (Adler \& Kwon, 2002; } \\
\text { Nahapiet \& Ghoshal, 1998); comblent le } \\
\text { vide institutionnel (Khanna \& Palepu, 1997, } \\
\text { 1998) }\end{array}$ \\
\hline $\begin{array}{l}\text { Effets du style des relations } \\
\text { sur la performance dans } \\
\text { les EF et les entreprises } \\
\text { indépendantes }\end{array}$ & $\begin{array}{l}\text { Hypothèse 3 : Plus importante dans } \\
\text { les EF; Les dirigeants d'EF forment } \\
\text { des relations plus personnelles avec } \\
\text { les employés; ils en reçoivent plus de } \\
\text { considération et de dévouement, ce qui } \\
\text { accroît la performance (Habbershon \& } \\
\text { Williams, 1999; Miller \& Lee, 2001) }\end{array}$ & $\begin{array}{l}\text { Hypothèse 6 : Plus développées dans les } \\
\text { EF. La stabilité, le pouvoir, les enjeux } \\
\text { de la famille attirent des partenaires plus } \\
\text { loyaux, des relations plus confiantes avec } \\
\text { les béfices (Bubolz, 2001; Miller \& Le } \\
\text { Breton-Miller, 2005). }\end{array}$ \\
\hline \hline
\end{tabular}


n'importe quel autre actionnaire, et où les décisions stratégiques sont prises par les divers membres d'une même famille.

Nous avons calculé l'OCE en utilisant les grilles de Lee \& Miller (1999) et de Miller \& Lee (2001). Il s'agit d'une échelle de Lickert à cinq échelons sur quatre items, pour évaluer l'engagement de l'entreprise quant au bien-être des employés, au paiement de salaires généreux et justes, à la satisfaction du personnel dans son travail et au partage des bénéfices (Voir Appendice B). Notre coefficient alpha de Cronbach pour cette mesure de l'OCE a été de 0,81. La fidélité inter-répondants, estimée par nos 35 dyades de directeurs (le directeur général et les sous-directeurs immédiats dans 35 entreprises choisies au hasard) a été de 0,78.

Nous avons mesuré l'importance des relations en prenant la valeur moyenne de deux composantes : celles de la direction (financement et statut politico-juridique) et celles liées à la technologie. Ces deux types de relations nous sont apparus comme les plus importantes pour ces entreprises. Les sociétés de haute technologie ont besoin d'un apport financier, juridique et technologique, et d'un apport de connaissances pour créer un produit, obtenir une licence d'exploitation, entreprendre sa production et innover capacité primordiale et source première d'avantage concurrentiel (Lee, 1998; Lee \& al., 2001). Nous avons mesuré les relations des équipes dirigeantes avec l'échelle de Likert à cinq échelons pour évaluer le degré (de très développé à faible) auquel l'organisation est engagée dans des relations durables avec des :

\section{1- agences gouvernementales \\ 2- investisseurs professionnels (VC) \\ 3- banques et compagnies d'assurances \\ 4- avocats \\ 5- experts et consultants financiers.}

Nous avons mesuré les relations technologiques avec trois échelles de Likert à 5 échelons pour évaluer la fréquence (de très rarement à très souvent) avec laquelle la compagnie entretient des relations durables avec des universités ou des instituts de recherche pour mener :

1- des actions associées de recherche ou de développement

2- des projets impliquant des transferts importants de technologie

3- des actions d'éducation et de formation.

Nos coefficients alpha de Cronbach pour les variables à deux dimensions ont été respectivement de 0,75 et de 0,87 , et de 0,82 pour la variable de relations à huit items. Le taux de fiabilité était de 0,78 et de 0,77 , et de 0,84 pour la variable à huit items.
Un second aspect des relations, leur extension géographique, a été mesuré par trois échelles qui allaient de local, à sous-régional, régional, national, international. Le coefficient alpha de Cronbach pour cette mesure a été de 0,70 et le taux de fiabilité de 0,76 .

Nous avons évalué la performance en utilisant les mesures de Gupta \& Govindarajan (1984). Notre échantillon comprenant des entreprises de domaines industriels différents, avec des objectifs et des critères de performance différents, nous avons dû trouver des mesures de performance relatives, multidimensionnelles et subjectives plutôt que de nous en tenir à un indicateur financier étroit. En outre, plus de $50 \%$ des répondants de l'échantillon, composé de sociétés privées, ont refusé de nous fournir des données financières. Nous avons demandé à nos interlocuteurs, à quel point la société avait atteint ses objectifs de rentabilité, de croissance, d'efficacité, de satisfaction du client, de gestion de l'emploi et de moral des employés. Le coefficient alpha de Cronbach a été ici de 0,84 et le taux de cohérence de 0,87 , ce qui montre une bonne corrélation entre les différentes analyses. Les limites de la mesure de la performance relèvent du fait que cette mesure ne reflète que partiellement et de façon imprécise la réussite financière et qu'elle est influencée par les préférences individuelles de chaque groupe.

\section{Analyses}

Nous avons classifié une entreprise comme EF lorsque la famille y est le plus gros actionnaire et qu'elle influence très activement les prises de décision. Pour tester les hypothèses 1 et 4, les tableaux 2, 3 et 4 présentent des études de comparaison de moyennes, des analyses de corrélation, des analyses de régression multiple, qui comparent entre EF et Entreprises Non Familiales l'importance du sens de la communauté et du réseau de relations. Le tableau 4 présente les résultats par âge de l'entreprise, taille et type d'activité (Jorrissen, Leveren, Martens, \& Reheul, 2005). Pour vérifier les hypothèses 2 et 5 concernant l'impact du sens de la communauté et du réseau de relations, nous avons effectué respectivement des analyses de corrélation et de régression hiérarchisée des tableaux 3 et 5 . À nouveau, nous avons analysé les régressions concernant l'âge et la taille des entreprises, leur activité, dans ce dernier cas utilisant des variables nominales. Les analyses complémentaires du tableau 6 ont été effectuées pour évaluer la généralité des hypothèses 2 et 5 dans des activités opérant en contexte plus ou moins instable. Finalement les hypothèses 3 et 6 sur le différentiel d'influence du sens de la communauté et du réseau de relations sur la performance dans les EF et les ENF ont été vérifiées par les termes d'interaction du tableau 5. Ces termes étaient un produit d'une variable nominale avec soit l'OCE soit le réseau de relations. Nous avons évalué l'importance des termes d'interaction selon la variance graduelle qu'ils expliquent sur celle des effets importants et des variables de contrôle. 
TABLEAU 2

Statistiques descriptives : ENF versus EF

\begin{tabular}{|c|c|c|c|c|c|c|c|}
\hline \multirow[t]{2}{*}{ Variables } & \multicolumn{2}{|c|}{$\begin{array}{l}\text { Échantillon total } \\
\quad N-170\end{array}$} & \multicolumn{2}{|c|}{$\begin{array}{c}\text { Entreprises } N F \\
\quad N=99\end{array}$} & \multicolumn{2}{|c|}{$\begin{array}{c}E F \\
N=71\end{array}$} & \multirow[t]{2}{*}{ Valeur $t$} \\
\hline & Moyenne & $\begin{array}{l}\text { Écart- } \\
\text { type }\end{array}$ & Moyenne & $\begin{array}{c}\text { Écart- } \\
\text { type }\end{array}$ & Moyenne & $\begin{array}{c}\text { Écart- } \\
\text { type }\end{array}$ & \\
\hline Taille & 28,34 & 44,56 & 23,49 & 30,46 & 32,06 & 53,81 & $-1,19$ \\
\hline$\hat{A} g e$ & 7,43 & 6,76 & 6,35 & 3,93 & 9,11 & 9,3 & $-2,34 * *$ \\
\hline Incertitude (fictive) & 0,79 & 0,41 & 0,83 & 0,38 & 0,73 & 0,45 & 1,47 \\
\hline Communauté OCE & 3,68 & 0,63 & 3,7 & 0,69 & 3,65 & 0,6 & 0,48 \\
\hline Relations & 2,79 & 0,68 & 2,7 & 0,73 & 2,79 & 0,6 & $-1,98 * *$ \\
\hline $\begin{array}{l}\text { Étendue géographique } \\
\text { des relations }\end{array}$ & 2,22 & 1,07 & 2,03 & 1,05 & 2,45 & 1,08 & $-2,56 * *$ \\
\hline Performance & 3,74 & 0,7 & 3,72 & 0,56 & 3,76 & 0,87 & $-0,31$ \\
\hline
\end{tabular}

TABLEAU 3

Corrélations de Pearson et alphas de Cronbach

\begin{tabular}{|c|c|c|c|c|c|c|c|}
\hline Variables & 1 & 2 & 3 & 4 & 5 & 6 & $\alpha$ \\
\hline Taille (nbre d'employés) & & & & & & & NA \\
\hline 2- Âge & $0,52 * * *$ & & & & & & NA \\
\hline 3 - Communauté (OCE) & $-0,07$ & $-0,1$ & & & & & 0,81 \\
\hline 4 - Relations & 0 & $-0,07$ & $0,17 * *$ & & & & 0,82 \\
\hline 5 - Étendue géographique & $0,25 * * *$ & $0,16 * *$ & 0,1 & $0,14^{*}$ & & & 0,7 \\
\hline 6 - Performance & 0,06 & $-0,01$ & $0,37 * * *$ & $0,20 * *$ & $0,21 * * *$ & & 0,84 \\
\hline 7 - Famille (fictive) & 0,1 & $0,20 * * *$ & $-0,04$ & $0,15^{* *}$ & $0,16 * *$ & 0,03 & NA \\
\hline
\end{tabular}

${ }^{*} \mathrm{p}<0,10 ; * * \mathrm{p}<0,05 ; * * * \mathrm{p}<0,01 ; \mathrm{N}=170$

\section{TABLEAU 4}

\section{Régression de la communauté et des relations sur une EF fictive et vérifications}

\begin{tabular}{|c|c|c|c|}
\hline Variable dépendante & Communauté & Relations & Étendue géographique \\
\hline Taille (nombre d'employés) & $-0,045$ & 0,143 & $0,210 * *$ \\
\hline$\hat{\text { Âge }}$ & $-0,127$ & $-0,188^{*}$ & 0,02 \\
\hline Ordinateurs & $-0,086$ & $-0,014$ & $-0,085$ \\
\hline Télécommunications & $-0,069$ & 0,059 & 0,004 \\
\hline Machines & 0,047 & $-0,116$ & $-0,031$ \\
\hline Famille (fictive) & $-0,048$ & $0,229 * * *$ & $0,134 *$ \\
\hline $\mathrm{N}$ & 170 & 170 & 170 \\
\hline Adj. $R^{2}$ & 0,001 & 0,048 & 0,047 \\
\hline $\mathrm{F}$ & 1,029 & $2,357 * *$ & $2,327 * *$ \\
\hline
\end{tabular}

$* \mathrm{p}<0,10 ; * * \mathrm{p}<0,05 ; * * * \mathrm{p}<0,01$ 


\section{TABLEAU 5}

\section{Régression de la performance sur la communauté et les relations}

\begin{tabular}{|c|c|c|c|}
\hline \multirow{2}{*}{$\begin{array}{l}\text { Variable dépendante } \\
\text { Taille (nombre d'employés) }\end{array}$} & \multicolumn{3}{|c|}{ Performance } \\
\hline & $0,147 * *$ & 0,031 & 0,063 \\
\hline Âge & $-0,041$ & $-0,066$ & $-0,12$ \\
\hline Ordinateurs & $-0,125^{*}$ & $-0,176^{*}$ & $-0,188^{*}$ \\
\hline Télécommunications & $-0,069$ & $-0,089$ & $-0,12$ \\
\hline Machines & $-0,055$ & 0,008 & $-0,019$ \\
\hline Famille (fictive) & 0,065 & $-0,042$ & 0,004 \\
\hline Communauté (0CE) & $0,655^{* * *}$ & & \\
\hline Relations & & $0,243 * * *$ & \\
\hline Étendue géographique des relations & & & $0,191 * *$ \\
\hline Famille x Communauté (OCE) & $0,154 * * *$ & & \\
\hline Famille et relations & & $0,143 *$ & \\
\hline Famille et extensions géographiques & & & $0,136^{*}$ \\
\hline $\mathrm{N}$ & 170 & 170 & 170 \\
\hline $\operatorname{Adj} R^{2}$ & 0,466 & 0,047 & 0,046 \\
\hline $\mathrm{F}$ & $18,86 * * *$ & $2,00 * *$ & $1,97 * *$ \\
\hline
\end{tabular}

TABLEAU 6

Régression de la performance sur la communauté et les relations : effet de l'incertitude de l'industrie

\begin{tabular}{|c|c|c|c|}
\hline \multirow{2}{*}{$\begin{array}{l}\text { Variable dépendante } \\
\text { Taille (nbre d'employés) }\end{array}$} & \multicolumn{3}{|c|}{ Performance } \\
\hline & $0,160 * *$ & 0,065 & 0,058 \\
\hline Âge & $-0,027$ & $-0,046$ & $-0,085$ \\
\hline Incertitude (fictive) & 0,018 & $-0,08$ & $-0,074$ \\
\hline Famille (fictive) & 0,072 & $-0,029$ & $-0,003$ \\
\hline Communauté (OCE) & $0,694 * * *$ & & \\
\hline Relations & & $0,221 * * *$ & \\
\hline Étendue géographique des relations & & & $0,226 * * *$ \\
\hline Indice d'incertitude $x$ communauté (OCE) & $-0,110^{*}$ & & \\
\hline Indice d'incertitude $\mathrm{x}$ relations & & 0,027 & \\
\hline Indice d'incertitude $\mathrm{x}$ étendue géographique & & & $-0,006$ \\
\hline $\mathrm{N}$ & 170 & 170 & 170 \\
\hline Adj. $R^{2}$ & 0,451 & 0,02 & 0,023 \\
\hline $\mathrm{F}$ & $23,444 * * *$ & 1,549 & 1,616 \\
\hline
\end{tabular}

$* \mathrm{p}<0,10 ; * * \mathrm{p}<0,05 ; * * * \mathrm{p}<0,01$. 


\section{Résultats}

\section{HYPOTHÈSES 1 ET 4 : LA COMMUNAUTÉ ET LE RÉSEAU DE RELATIONS DANS LES EF ET LES ENF}

Les comparaisons de moyennes du tableau 1 montrent que l'OCE n'est pas significativement différent dans les EF et les ENF, mais que les relations sont plus développées dans les EF. Pour déterminer si ces résultats étaient influencés par les différences d'âge, de taille et d'industrie (Jorissen et al., 2005) entre EF et ENF, nous avons effectué les analyses de régression multiple du tableau 4, incorporant sur les variables d'une firme nominale ces différences potentielles comme variable de contrôle quand régressaient le sens de la communauté et les réseaux de relations $(1=\mathrm{F}, \mathrm{O}=\mathrm{NF})$. Les résultats confirment ceux du tableau 1. L'hypothèse 1 n'est donc pas confirmée, mais la 4 l'est. Même si nos EF de haute technologie ont davantage tendance à nouer des relations avec des partenaires extérieurs que les ENF, elles ne sont pas à même en général de construire une communauté interne qui traitent mieux leurs employés que les firmes homologues non familiales - peut-être parce que ces dernières sont aussi très attentives à leur personnel.

\section{HypothèSES 2 et 5 : L'INFLUENCE de la COMMUNAUTÉ ET DES RELATIONS SUR LA PERFORMANCE.}

La grille de corrélations du tableau 3 montre que la communauté et les relations ont des corrélations positives avec la performance, soutenant les hypothèses 2 et 5 respectivement. Constatation plus importante, ces relations sont confirmées par les analyses de régression multiple du tableau 5. Pour nos sociétés de haute technologie, la communauté et les relations contribuent également à la performance généralement définie. L'extension géographique des relations produit le même effet. Ces résultats sont en accord avec l'idée que, pour les industries de haute technologie des marchés émergents, relations et liens particuliers sont sources d'accès aux ressources nécessaires à l'innovation et à la bonne performance (Lee, 1998; Lee \& Chan, 1999; Lee \& al., 2001).

Afin d'établir la solidité de ces relations à différents niveaux d'instabilité industrielle, nous avons défini une nouvelle variable nominale. La variable avait une valeur de 1 pour les industries les plus exposées d'informatique et de télécommunications, et une valeur de 0 pour les industries les plus exposées de mécanique. Cette division se basait sur l'analyse par Lee (1998) de l'instabilité pour les firmes de haute technologie des marchés émergents. La performance de l'entreprise régressait contre les termes d'interaction qui étaient le produit de la variable nominale d'instabilité et de communauté, de relations, et de leur étendue géographique. Comme le montre le tableau 6, ces résultats d'interaction n'étaient pas liés à la performance, pas plus que ne l'était la variable nominale d'instabilité. Donc la validité des hypothèses 2 et 5 ne varie pas matériellement parmi les firmes de notre échantillon. Nous hésitons cependant à appliquer ces résultats aux firmes opérant en milieu stable, car toutes nos entreprises appartenaient au secteur de haute technologie, et toutes opéraient dans un contexte très instable.

\section{Hypothèses 3 eT 6 : L'INFLUENCE de la COMMUNAUTÉ ET DES RELATIONS SUR LA PERFORMANCE DANS LES EF ET LES ENF.}

Les analyses de régression du tableau 5 prédisent la performance. Elles confirment respectivement les interactions importantes entre les variables nominales pour le statut de l'EF et la communauté, pour les relations et leur extension géographique. Les analyses d'interaction permettent d'apprécier si les EF étaient mieux à même de bénéficier d'un taux plus élevé de communauté ou de relations que les ENF. Les résultats confirment très nettement l'interaction entre famille et communauté, mais moins nettement les deux interactions entre famille et relations. En outre les valeurs $\mathrm{F}$ pour les changements en $\mathrm{R}^{2}$ dérivés de l'addition des termes d'interaction pour le modèle complet étaient de 7,068, 3,338 et 2,934. Ces coefficients sont significatifs audelà des niveaux $0,01,0,10$. Ces résultats confirment donc clairement l'hypothèse 3 et un peu moins l'hypothèse 6 . Il apparaît donc que la communauté, les relations et des liens géographiquement étendus sont plus utiles dans les EF que dans les autres.

\section{Discussion, implications et limites}

Ces résultats confirment significativement la plupart de nos hypothèses. L'investissement dans la communauté et les relations ont donc un rapport évident avec le succès dans nos marchés émergents de haute technologie, et ce double investissement est plus bénéfique dans les EF que les autres. Les relations sont aussi plus développées dans les EF. La grande surprise a été de constater que l'OCE n'est pas plus développé dans les EF que dans les autres firmes, ce qui peut expliquer pourquoi la performance ne varie pas de façon significative entre EF et ENF. Peut-être que dans ces milieux de haute technologie, les employés talentueux constituant clairement une catégorie essentielle, toutes les entreprises essayent de bien les traiter - EF et ENF tout autant. Autre raison à cet échec des EF de notre échantillon à obtenir de meilleure performance, les défauts propres à certaines d'entre elles : conflits familiaux, népotisme, et attachement à la tradition particulièrement nocif en milieu compétitif et instable. Ces défauts peuvent neutraliser tous les aspects positifs de la communauté et des relations. Enfin, nos ENF sont aussi des entreprises de haute technologie, donc des sociétés dynamiques capables d'éviter les pièges bureaucratiques en comparaison à d'autres ENF provenant de secteurs plus stables

On peut déduire plusieurs choses de cette recherche pour l'industrie de la haute technologie. La première, c'est 
que l'OCE - une dimension humaine - est un facteur de performance aussi important pour les EF que pour les ENF dans l'industrie de haute technologie. Plus spécifiquement, les organisations qui nouent des relations affectives étroites avec leurs employés en se souciant leur bien-être, en récoltent les fruits, mais ceci surtout dans les EF, où la motivation, le dévouement et la coopération entre les employés peuvent constituer une ressource précieuse. Il en va de même pour la formation de liens avec des fournisseurs extérieurs d'expertise, de ressources humaines et financier, auxquels les EF ont plus recours que les autres. Enfin, ceci confirme les thèses de Khanna et Palepu (1997, 1999 et 2006) voulant que la formation d'une communauté et la création de relations contribuent à pallier le vide institutionnel des économies émergentes.

\section{LIMITES DE LA RECHERCHE ET NOUVELLES DIRECTIONS}

Il faut noter les limites de notre recherche. D'abord, ces résultats s'appliquent surtout aux petites compagnies de haute technologie dans des environnements instables. Il est impossible de dire si nos résultats se confirmeraient dans des contextes plus stables, ou pour des compagnies plus grosses. Et si nos conclusions sur la Corée du Sud peuvent se vérifier dans d'autres économies émergentes aux institutions précaires, il conviendrait de voir si cela vaut pour le monde développé. Nous espérons que notre étude suscitera d'autres recherches sur les EF. On pourrait par exemple étudier de façon plus détaillée comment les EF diffèrent des autres par leurs comportements, leurs stratégies et leurs performances. Il pourrait aussi être utile de mener cette même étude dans d'autres pays et industries pour généraliser ou limiter les résultats.

\section{Conclusion}

Il peut sembler paradoxal que ces caractéristiques des EF, beaucoup critiquées dans la littérature sur le management et la direction d'entreprise, soient justement celles qui leur permettent, ainsi qu'à d'autres, d'obtenir de bonnes performances en haute technologie dans des marchés émergents.

Les reproches faits aux EF - leurs relations affectives, collectives, communautaires avec leurs employés, et leurs relations durables et étroites avec leurs partenaires extérieurs - semblent justement être les qualités qui contribuent à leur succès. En outre, cet avantage semble s'appliquer non pas aux environnements établis et calmes, mais aux plus concurrentiels et aux plus évolutifs. Ce mode relationnel - opposé au transactionnel - contribue aux bonnes performances des EF, et, de façon moins évidente, à celle des autres. On peut donc en déduire que les relations étroites qu'entretiennent les EF avec leur environnement leur donnent un avantage pour surmonter le vide institutionnel propre à ces marchés émergents (Khanna \& Palepu, 1997, 1999). Les dirigeants travaillant dans ce contexte et les poli- tiques au pouvoir feraient bien de remarquer l'importance des EF dans la haute technologie et d'étudier ce qu'ils peuvent en apprendre pour améliorer la compétitivité de leurs entreprises.

\section{Bibliographie}

Adler, P. S., \& Kwon, S. W. 2002. Social capital: Prospects for a new concept. Academy of Management Review, 27(1):17-40.

Allouche, J., \& Amann, B. 1997. Le retour du capitalisme familial. L'expansion: Management Review, 85(1): 92-99.

Anderson, R. C., \& ReEB, D. 2003. Founding-family ownership and firm performance: Evidence from the S\&P 500. Journal of Finance, 58(3): 1301-1328.

Arregle, J. L., Hitt, M., Sirmon, D., \& Very, P. 2007. The development of organizational social capital: Attributes of family firms. Journal of Management Studies, 44(1): 73-95.

Barney, J. B., \& HANSEN, M. H. 1994. Trustworthiness as a source of competitive advantage. Strategic Management Journal, 15(Winter Special Issue): 175-190.

Beehr, T., Drexler, J., \& Faulkner, S. 1997. Working in small family businesses: Empirical comparisons to non-family businesses. Journal of Organizational Behavior, 18(3): 297-312.

Bertrand, M., \& Schoar, A. 2006. The role of family in family firms. Journal of Economic Perspectives, 20(2): 73-96.

Bubolz, M. 2001. Family as source, user and builder of social capital. Journal of Socio-Economics, 30(1): 129-131.

CARney, M. 2005. Corporate governance and competitive advantage in family-controlled firms. Entrepreneurship Theory and Practice, 29(2): 249-265.

Chandler, A. D. 1990. Scale and scope. Cambridge, MA: Harvard University Press.

CHOI, J. 2004. Transformation of Korean HRM based on confucian values. Seoul Journal of Business, 10(1): 1-26.

Collins, J. 1995. Built to last. New York: HarperBusiness.

Cooper, A. C., Willard, G. E., \& Woo, G. 1986. Strategies of high performing new and small firms: A reexamination of the niche concept. Journal of Business Venturing, 1(2): 247-260.

Davis, S., \& MeYer, C. 1998. Blur. New York: Warner Books.

Donckels, R., \& FröHLICH, E. 1991. Are family businesses really different? European experiences from STRATOS. Family Business Review, 4(2): 149-160.

Duchesneau, D. A., \& Gartner, W. 1990. A profile of new venture success and failure in an emerging industry. Journal of Business Venturing, 5(3): 297-312.

Dyer Jr., W. G. 1986. Cultural change in family firms: Anticipating and managing business and family transitions. San Francisco:Jossey-Bass.

Eisenberger, R., Fasolo, P., \& Davis-Lamastro, V. 1990. Perceived organizational support and employee diligence, commitment and innovation. Journal of Applied Psychology, 75(1): 51-59.

Eisenberger, R., Huntington, R., Hutchison, R., \& Sowa, D. 1986. Perceived organizational support. Journal of Applied Psychology, 71(4): 500-507. 
Galbraith, J. 2000. Designing the global corporation. San Francisco: Jossey Bass.

Gersick, K. E., Davis, J., Hampton, M., \& Lansberg, I. 1997. Generation to generation: Life cycles of the family business. Boston, MA: Harvard Business School Press.

GomeZ-Mejia, L., Haynes, K., NuñEz-Nickel, M., Jacobson, K., \& Moyano-Fuentes, J. 2007. Family owned firms: Risk loving or risk averse. Administrative Science Quarterly, 52(1): 106-137.

Gomez-Mejia, L., Nuñez-Nickel, M., \& Gutierrez, I. 2001. The role of family ties in agency contracts. Academy of Management Journal, 44(1): 81-95.

Grant, R. 1996. Toward a knowledge-based theory of the firm. Strategic Management Journal, 17(Special Issue): 109-122.

Gupta, A. K., \& Govindarajan, V. 1984. Business unit strategy, managerial characteristics, and business unit effectiveness at strategy implementation. Academy of Management Journal, 27(1): 25-41.

Guzzo, R., \& Аввотт, S. 1990. Family firms as utopian organizations. Family Business Review, 3(1): 23-33.

Habbershon, T. G., \& Williams, M. L. 1999. A resource-based framework for assessing the strategic advantages of family firms. Family Business Review, 12(1): 1-26.

Hagel, J., \& Singer, J. 1999. Net worth. Boston, MA: Harvard Business School Press.

JACOBS, M. T. 1991. Short-term America. Boston, MA: Harvard Business School Press.

JAMES, H. S. 1999. Owner as manager, extended horizons and the family firm. International Journal of the Economics of Business, 6(1): 41-55.

Jorissen, A., Leveren, E., Martens, R., \& Reheul, A. M. 2005. Real versus sample-based differences in comparative family business research. Family Business Review, 18(3): 229-246.

Khanna, T., \& Palepu, K. 1997. Why focused strategies may be wrong for emerging markets. Harvard Business Review, 75(4): $41-51$.

Khanna, T., \& Palepu, K. 1999. The right way to structure conglomerates in emerging markets. Harvard Business Review, 77(4): 125-134.

Khanna, T., \& Palepu, K. 2000. Is group affiliation profitable in emerging markets?: An analysis of diversified Indian business groups. Journal of Finance, 55(4): 867-891.

Khanna, T., \& Palepu, K. 2006. Emerging giants? Harvard Business Review, 84(4): 60-69.

Khanna, T., \& Rivkin, J. W. 2000. Estimating the performance effects of business groups in emerging markets. Strategic Management Journal, 22(1): 45-74.

KocH, C. 2007. The science of success. New York: Wiley.

LANDES, D. 1949. French entrepreneurship and industrial growth in the nineteenth century. Journal of Economic History, 9(1): $45-61$.

LaPorta, R., Lopes-de-Silanes, F., \& Shleifer, A. 1999. Corporate ownership around the world. Journal of Finance, 54(3): 471-517.
LAZONICK, W. 1986. The cotton industry. In B. Erbaum \& W. Lazonick (Eds), The decline of the British economy: 18-50. Oxford: Oxford University Press.

LEE, J. 1998. Characteristics of successful high-technology venture companies. The Korean Venture Management Review, 1(1): 101-128.

LeE, J., \& Chang, S. 1999. Characteristics of successful high-tech ventures. The Korean Small Business Review, 21(1): 105-133.

LEE, J., \& LEE, M. 1994. Korean management in global competition. Seoul: Gim-Young Sa.

Lee, J., \& Miller, D. 1999. People matter: Commitment to employees, strategy and performance in Korean firms. Strategic Management Journal, 20(4): 579-593.

Lee, C., Lee, K., \& Pennings, J. 2001. Internal capabilities, external networks, and performance: A study of technology-based ventures. Strategic Management Journal, 22(4): 615-640.

Mackie, R. 2001. Family ownership and business survival: Kirkcaldy, 1870-1970. Business History, 43(1): 1-32.

Miller, D. 2003. An asymmetry-based view of advantage: Towards an attainable sustainability. Strategic Management Journal, 24(10): 961-976.

Miller, D., \& Lee, J. 2001. The people make the process: Commitment to employees, decision making, and performance. Journal of Management, 27(2): 163-189.

Miller, D., \& Le Breton-Miller, I. 2005. Managing for the long run: Lessons in competitive advantage from great family business. Boston, MA: Harvard Business School Press.

Miller, D., Steier, L., \& Le Breton-Miller, I. 2003. Lost in time: Intergenerational succession, change, and failure in family business. Journal of Business Venturing, 18(3): 513-531.

Miller, D., Le Breton-Miller, I., Lester, R., \& Cannella, A. 2007. Are family firms really superior performers? Journal of Corporate Finance, 13(5): 829-858.

Miller, D., Le Breton-Miller, I., \& Scholnick, B. 2008. Stewardship versus stagnation: An empirical comparison of small family vs non-family businesses. Journal of Management Studies, 41(1): 50-78.

Moorman, S., Blakely, G., \& Niehoff, B. 1998. Does perceived organizational support mediate the relationship between procedural justice and organizational citizenship behavior? Academy of Management Journal, 41(2): 351-357.

Morck, R., Wolfenzon, D., \& Yeung, B. 2005. Corporate governance, economic entrenchment, and growth. Journal of Economic Literature, 53(3): 655-720.

Nahapiet, J., \& GHoshal, S. 1998. Social capital, intellectual capital and the organizational advantage. Academy of Management Review, 23(2): 242-266.

NAM, Y. H. 2002. A study on the characteristics of Korean family businesses. The Korean Small Business Review, 24(4): 201224.

NonAKA, I. 1994. A dynamic theory of organizational knowledge creation. Organization Science, 5(1): 14-37.

NonaKa, I. 1995. The knowledge-creating company. New York: Oxford University Press.

O'Reilly, C., \& Chatman, J. 1986. Organizational commitment and psychological attachment: The effects of compli- 
ance, identification, and internalization on prosocial behavior. Journal of Applied Psychology, 71(3): 492-499.

ORPEN, C. 1995. The effects of exchange ideology on the relationship between perceived organizational support and job performance. Journal of Social Psychology, 78(3): 569-583.

PARK, G. D. 1982. A study on the family business of Korea. Korean Management Review, 11(1): 39-60.

Pfeffer, J., \& SAlANCIK, G. 1978. The external control of organizations. New York: Harper \& Row.

REID, R., \& HARris, R. 2002. The determinants of training in SMEs in Northern Ireland. Education \& Training, 44(1): 8-9.

SAXTON, T. 1997. The effects of partner and relationship characteristics on alliance outcomes. Academy of Management Journal, 40(2): 443-461.

Schulze, W. S., Lubatkin, M., Dino, R., \& Buchholtz, A. 2001. Agency relationships in family firms: Theory and evidence. Organization Science, 12(1): 99-116.

Shanker, M. C., \& Astrachan, J. H. 1996. Myths and realities: Family business' contribution to the US economy: A framework for assessing family business statistics. Family Business Review, 9(2): 107-123.

SHIN, Y. K. 1993. Korean management: Present and future. Seoul: Bak-Young Sa.

Singell, L. 1997. Nepotism, discrimination, and the persistence of utility-maximizing, owner-operated firms. Southern Economics Journal, 63(4): 894-920.

Sirmon, D., \& HitT, M. 2003. Managing resources: Linking unique resources, management, and wealth creation in family firms. Entrepreneurship Theory and Practice, 27(3): 339-358.

Villalonga, B., \& Amit, R. 2006. How do family ownership, control and management affect firm value? Journal of Financial Economics, 80(3): 385-415.

WARD, J. 2004. Perpetuating the family business. Marietta: Family Enterprise Publishers.

Williamson, O. E. 1999. Strategy research: Governance and competence perspectives. Strategic Management Journal, 20(7): 1087-1108.

Zahra, S. A., Hayton, J., \& Salvato, C. 2004. Entrepreneurship in family vs non-family firms: A resource-based analysis of the effect of organizational culture. Entrepreneurship Theory and Practice, 28(4): 363-381. 


\section{APPENDICE A}

Tableau A1 Caractéristiques des sociétés répondantes

\begin{tabular}{|c|c|c|c|c|c|c|c|}
\hline \multirow{3}{*}{$\begin{array}{l}\text { Item } \\
\text { Age de la société }\end{array}$} & \multirow{3}{*}{$\begin{array}{l}\text { Catégorie } \\
1 \text { à } 2 \text { ans }\end{array}$} & \multicolumn{2}{|c|}{ Total } & \multicolumn{2}{|c|}{$E N F$} & \multicolumn{2}{|c|}{$E F$} \\
\hline & & \multicolumn{2}{|c|}{ Nombre en $\%$} & \multicolumn{2}{|c|}{ Nombre en $\%$} & \multicolumn{2}{|c|}{ Nombre en $\%$} \\
\hline & & 13 & 7,7 & 9 & 5,3 & 4 & 2,4 \\
\hline & 3 à 4 ans & 71 & 42,0 & 41 & 24,3 & 30 & 17,8 \\
\hline & 5 à 6 ans & 46 & 27,2 & 29 & 17,2 & 17 & 10,1 \\
\hline & 7 ans et plus & 39 & 23,1 & 20 & 11,8 & 19 & 11,2 \\
\hline \multirow[t]{4}{*}{ Nombre d'employés } & Moins de 11 & 78 & 47 & 45 & 27,1 & 33 & 19,9 \\
\hline & Entre 11 et 20 & 34 & 20,5 & 20 & 12,0 & 14 & 8,4 \\
\hline & Entre 21 et 40 & 25 & 15,1 & 18 & 10,8 & 7 & 4,2 \\
\hline & 41 et plus & 29 & 17,5 & 15 & 9 & 14 & 8,4 \\
\hline \multirow[t]{4}{*}{ Industrie } & Ordinateurs & 52 & 30,6 & 33 & 19,4 & 19 & 11,2 \\
\hline & Télécom & 40 & 23,5 & 25 & 14,7 & 15 & 8,8 \\
\hline & Machines & 36 & 21,22 & 17 & 10,0 & 19 & 11,2 \\
\hline & Électronique & 42 & 24,7 & 24 & 14,1 & 18 & 10,6 \\
\hline $\mathrm{N}$ valid & & 170 & 100 & 99 & 58,2 & 71 & 41,8 \\
\hline
\end{tabular}




\section{APPENDICE B}

Tableau B1 items d'échelle du questionnaire

\section{Communauté (OCE)}

Évaluez à quel degré vous êtes d'accord avec ce qui suit :

Pas d'accord

L'organisation se soucie réellement du bien être de ses employés. $3.56(0.72)^{\mathrm{a}}$

La société veille scrupuleusement à payer chacun selon son mérite. $3.87(0,68)$

La société prend à cœur la satisfaction générale de ses employés dans leur travail. 3.53 (0.77)

Si la société faisait plus de bénéfices, elle les partagerait $3.79(0,69)$

\begin{tabular}{lllll}
1 & 2 & 3 & 4 & 5 \\
1 & 2 & 3 & 4 & 5 \\
1 & 2 & 3 & 4 & 5 \\
1 & 2 & 3 & 4 & 5 \\
\hline
\end{tabular}

\section{Relations entretenues par la direction}

Évaluez la fréquence des interactions de votre société avec les organismes suivants pour construire de fortes relations directoriales

Très rarement

Très souvent

Les agences gouvernementales

$2,69(1,04)$

$\begin{array}{lllll}1 & 2 & 3 & 4 & 5\end{array}$

Les experts financiers et les consultants

$2,67(0,93)$

1

2

3

4

5

Les avocats

$2,08(0,94)$

1

2

3

4

5

Les spécialistes de «capital-risque»

$2,04(0,89)$

1

2

3

4

5

Banques et assurance $2,54(0,94)$
2

3

4

5

\section{Relations technologiques}

Évaluez la fréquence avec laquelle vous coopérez durablement avec des universités ou des instituts de recherches.

Très rarement

Très souvent

Recherche et actions de développement en commun 2,99 (1.34)

Transferts techniques et technologiques et échanges d'information. 3,06 $(1,22)$

Éducation et formation $2,79(1,13)$

$\begin{array}{llllll}1 & 2 & 3 & 4 & 5 \\ 1 & 2 & 3 & 4 & 5 \\ 1 & 2 & 3 & 4 & 5\end{array}$


Tableau B1 (suite)

Étendue géographique des relations : de local à régional à national à international

Indiquez les régions de vos transactions les plus importantes

Partenaires d'affaires (1) À l'intérieur de la zone de high-tech existante et de Daegu

$2,36(1,44)$

(2) Province de Kyungpook

(3) Province de Yongnam et région de Séoul

(4) Autre région du pays

(5) Étranger

Clients

$2,31(1,43)$

(1) À l'intérieur de la zone de high-tech existante et de Daegu

(2) Province de Kyungpook

(3) Province de Yongnam et région de Séoul

(4) Autre région du pays

(5) Étranger

Fournisseurs

(1) À l'intérieur de la zone de high-tech existante et de Daegu

$1,98(1,41)$

(2) Province de Kyungpook

(3) Province de Yongnam et région de Séoul

(4) Autre région du pays

(5) Étranger

\section{Performance}

Comment l'entreprise a-t-elle atteint les objectifs suivants face à ses concurrents principaux ces 3 dernières années?

\begin{tabular}{|c|c|c|c|c|c|}
\hline & Très mal & & & & Très bien \\
\hline $\begin{array}{l}\text { Rentabilité obtenue } \\
3,50(0,84)\end{array}$ & 1 & 2 & 3 & 4 & 5 \\
\hline $\begin{array}{l}\text { Croissance de l'entreprise et augmentation des commandes } \\
3,92(0,78)\end{array}$ & 1 & 2 & 3 & 4 & 5 \\
\hline $\begin{array}{l}\text { Amélioration de la fidélité des clients } \\
4,08(0,72)\end{array}$ & 1 & 2 & 3 & 4 & 5 \\
\hline $\begin{array}{l}\text { Amélioration de l'efficacité interne } \\
3,77(0,72)\end{array}$ & 1 & 2 & 3 & 4 & 5 \\
\hline $\begin{array}{l}\text { Rotation des employés } \\
3,68(0,89)\end{array}$ & 1 & 2 & 3 & 4 & 5 \\
\hline $\begin{array}{l}\text { Moral des employés } \\
3,62(0,75)\end{array}$ & 1 & 2 & 3 & 4 & 5 \\
\hline
\end{tabular}

\section{Entreprise familiale}

Nous avons désigné comme familiale une entreprise où il était répondu OUI aux deux items suivants

1. La famille possède plus de parts que tout autre actionnaire

Oui ( )

Non ( )

2. Les décisions stratégiques sont très influencées par les membres de la famille

Oui ( )

Non ( )

${ }^{a}$ Les chiffres sont les moyennes d'items et les écarts types 